\title{
Les Monogènes Capsalinae \\ parasites des thons de l'Atlantique tropical oriental
}

\author{
par Jean BUSSIERAS
}

Institut des Sciences et Médecine Vétérinaires, Université de Dakar, Sénégal

\begin{abstract}
Résumé
Les nombreux Capsalinae (Monogenea: Capsalidae) récoltés sur les thons Thunnus obesus et Thunnus albacares de l'Atlantique tropical oriental, appartiennent à 4 espèces, dont 2 nouvelles : Caballerocotyla verrucosa n. sp., C. abidjani Bussiéras et BaudinLaurencin, 1970, C. pseudomagronum n. sp. et C. klawei Stunkard, 1962.

L'auteur signale que chez $C$. abidjani le pénis présente une véritable évagination, fait remarquable chez un Monogène.

Enfin est donnée une clé de diagnose des différentes espèces du genre Caballerocotyla, en désignant les poissons-hôtes selon la terminologie actuellement en usage.
\end{abstract}

\section{Summary}

Many Capsalinae (Monogenea: Capsalidae) were collected in tropical Eastern Atlantic on tunas of the species Thunnus obesus and Thunnus albacares. They belong to 4 species, among them 2 are new: Caballerocotyla verrucosa $\mathrm{n}$. sp., C. abidjani Bussiéras et Baudin-Laurencin, 1970, C. pseudomagronum n. sp. and $C$. klawei Stunkard, 1962.

The author observed that, in $C$. abidjani, the penis shows a true evagination, a noteworthy fact in a Monogenea.

At the end is given a key to the different species of the genus Caballerocotyla, in which the hosts-fishes are mentioned according to the present day classification. 
Chaque année, des quantités considérables de thons sont débarquées à Dakar ; l'examen de plus d'une centaine de ces poissons a permis de récolter de nombreux Monogènes du genre Caballerocotyla Price, 1960 (fam. Capsalidae Baird, 1853, s/fam. Capsalinae Johnston, 1929).

Les poissons examinés appartiennent à deux espèces : 1788) ;

- le thon albacore, ou thon à nageoires jaunes, Thunnus albacares (Bonnaterre,

- le patudo, ou thon obèse, Thunnus obesus (Lowe, 1839).

Tous ont été pêchés d'avril à juin 1971 , entre $8^{\circ}$ et $13^{\circ}$ de latitude nord, $16^{\circ}$ et $19^{\circ}$ de longitude ouest.

Les parasites récoltés peuvent être placés dans 4 espèzes, dont 2 nous paraissent nouvelles.

\section{CABALLEROCOTYLA VERRUCOSA n. sp.}

\section{Hôtes et HABITAT :}

Thunnus obesus: vers fixés soit dans la cavité buccale, sur la langue ou le palais, soit sur la face interne des opercules, soit très rarement sur les branchies elles-mêmes.

Thunnus albacares: vers toujours fixés dans la cavité buccale (langue ou palais).

MATÉRIEL ÉTUdié :

Plusieurs dizaines d'exemplaires, montés in toto après coloration (carmin de Sémichon, trichrome de Gomori, etc...).

\section{DESCRIPTION :}

Vers aplatis, à contuur ovalaire (fig. 1) ; longueur $8-11 \mathrm{~mm}$, largeur 3,5-6 mm ; la plus grande largeur se situe généralement un peu en arrière de la mi-longueur; le bord postérieur du corps est régulièrement arrondi. Par exception, certains exemplaires (fig. 2) ont un aspect cordiforme, relativement plus courts pour une largeur équivalente, et avec le bord postérieur profondément échancré ; le fait que tous les autres caractères anatomiques sont rigoureusement identiques conduit à admettre qu'il s'agit là d'exemplaires anormalement contractés mais appartenant bien à la même espèce.

A l'extrémité antérieure, les deux prohapteurs ont une forme circulaire, d'un diamètre de 0,73 à $1,05 \mathrm{~mm}$; ils sont distants de 0,36 à $0,63 \mathrm{~mm}$ l'un de l'autre.

L'opisthapteur (fig. 3), fixé à l'extrémité postérieure du corps, a un contour légèrement ovalaire ; grand axe 1,91-2,93 $\mathrm{mm}$, petit axe 1,73-2,56 $\mathrm{mm}$ (membrane périphérique non comprise). La membrane périphérique, formée de 100 à 110 festons, a une largeur de 0,24 à $0,32 \mathrm{~mm}$. L'opisthapteur est du type habituel dans le genre Caballerocotyla; il présente un heptagone central de type ouvert, et sept côtes rayonnantes ; il est surtout remarquable par la présence, sur toute sa face ventrale, de très nombreuses papilles; les papilles situées sur les côtes donnent à celles-ci un aspect verruqueux 


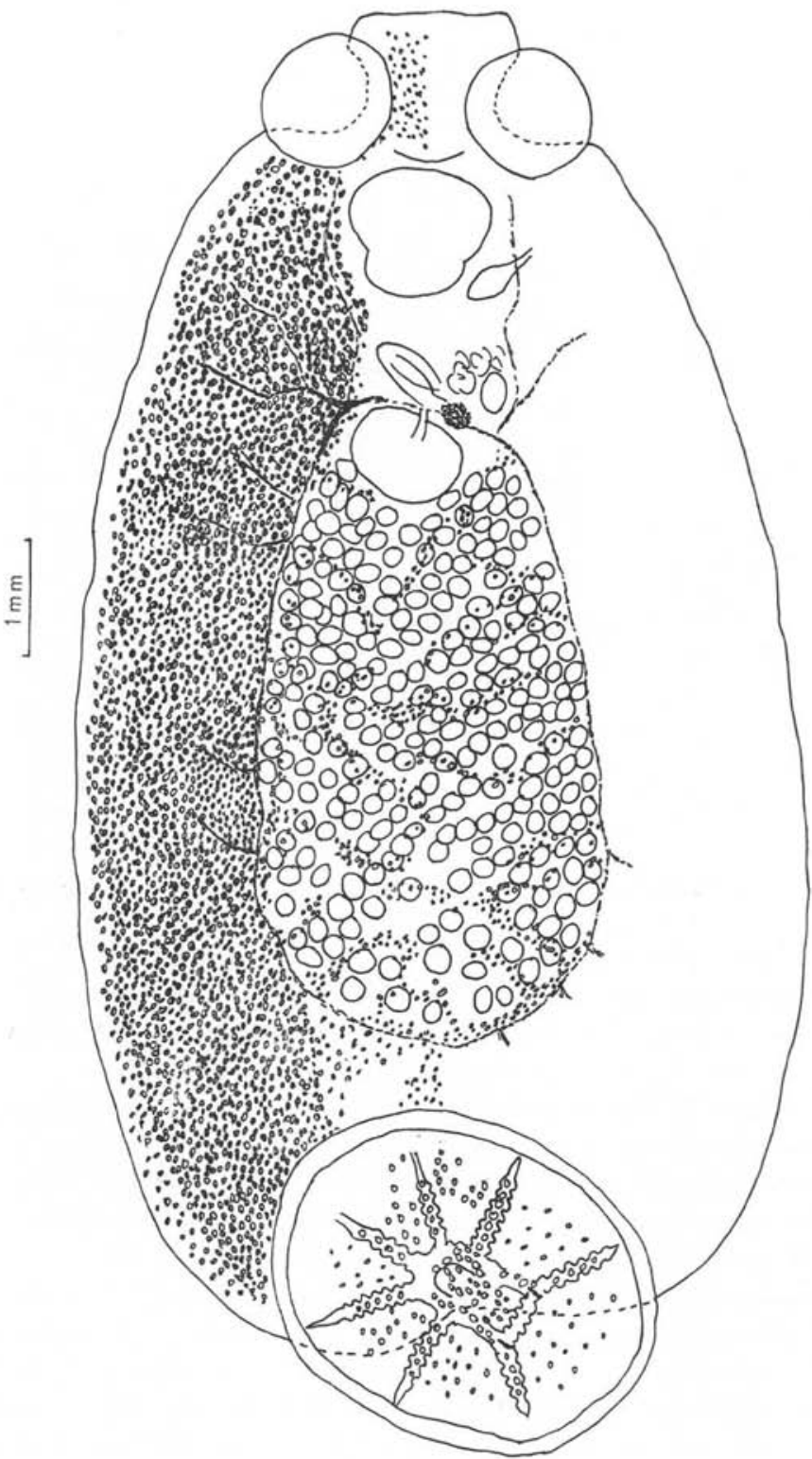

Fig. 1. - Caballerocotyla verrucosa, vue ventrale 


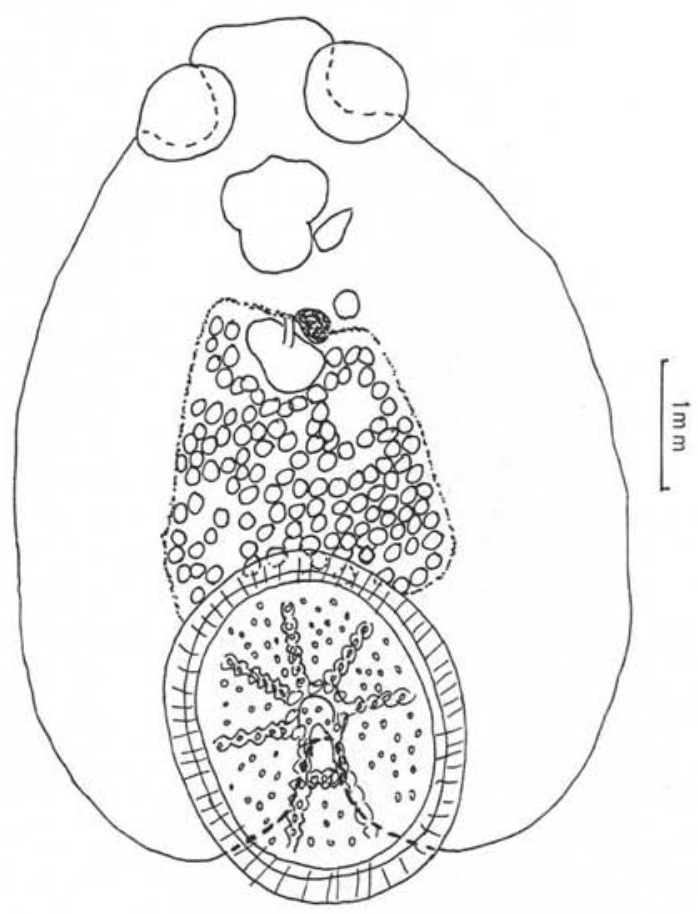

Fig. 2. - Caballerocotyla verrucosa, spécimen à bord postérieur échancré

très caractéristique. L'opisthapteur, en outre, porte deux ancres de forme simple, rectiligne ou légèrement incurvée, de 0,12 à $0,20 \mathrm{~mm}$ de long (*); enfin, on observe assez facilement 14 petits crochets marginaux (longueur 15 à $23 \mu$ ), disposés régulièrement par rapport aux festons de la membrane périphérique (fig. 4).

La face dorsale du ver ne présente pas de rangées de grosses épines, mais seulement de chaque côté une petite zone marginale, qui fait immédiatement suite à chaque prohapteur, et qui est recouverte de nombreuses petites épines transparentes, peu visibles, de 8 à $11 \mu$ de long, à une seule pointe ; ces épines sont disposées selon de courtes rangées transversales, assez irrégulières, de trois ou quatre éléments (fig. 5).

L'appareil digestif s'ouvre par une bouche située de 1,02 à 1,28 mm de l'extrémité antérieure ; le pharynx présente une constriction bien marquée ; sa longueur est de 0,77 à $0,93 \mathrm{~mm}$, sa largeur de 0,79 à $1,10 \mathrm{~mm}$ pour la partie antérieure, de 0,53 à $0,69 \mathrm{~mm}$ pour la partie postérieure ; il porte sur son bord antérieur de longues papilles transparentes, dirigées vers l'avant (fig. 6). Les deux branches de l'intestin, réunies postérieurement en anneau, sont peu visibles.

(*) Les ancres des Caballerocotyla n'étant généralement pas orientées dans un plan horizontal, il est nécessaire de calculer une correction pour obtenir leur longueur réelle. 


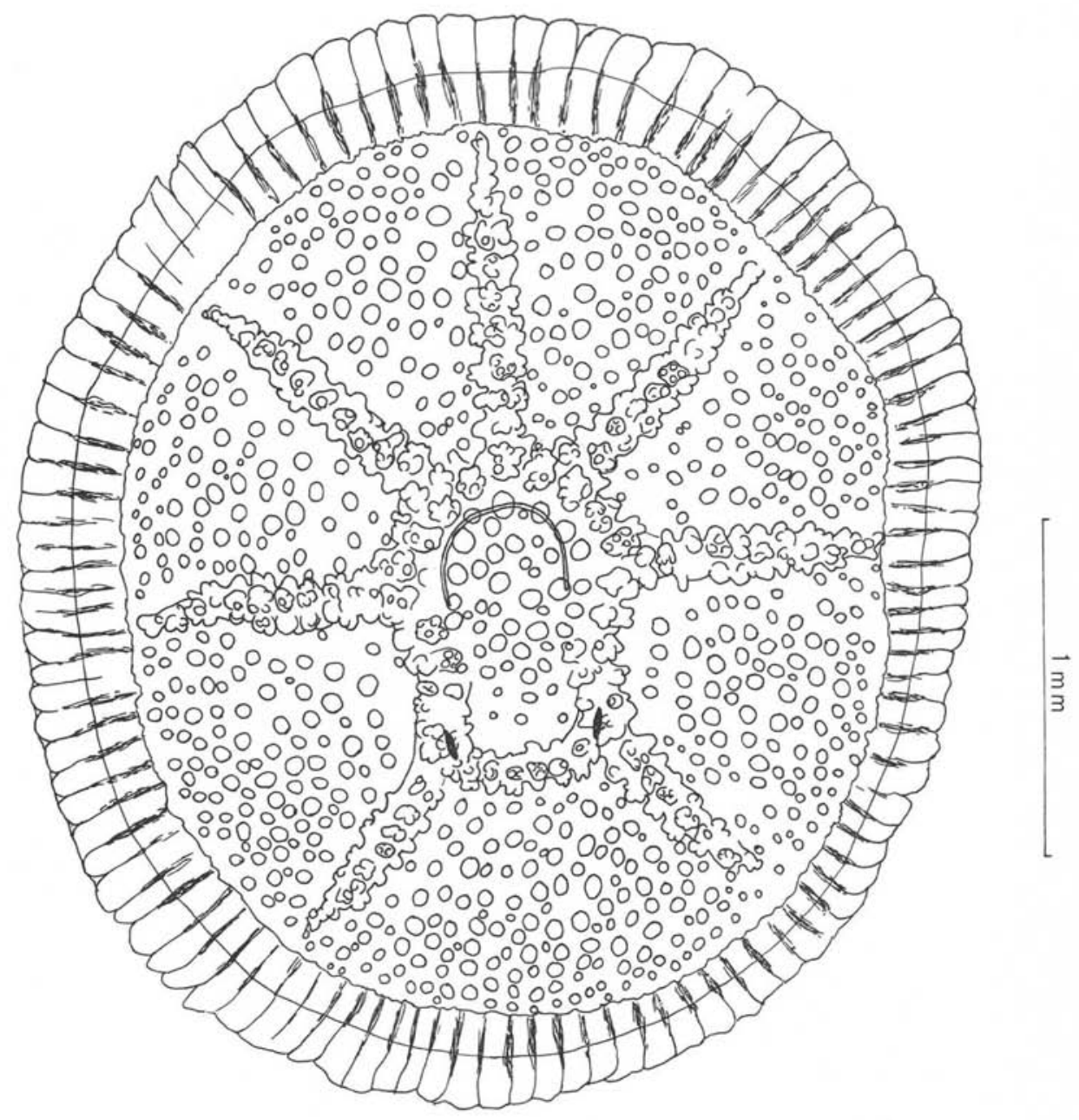

Fig. 3. - Caballerocotyla verrucosa, opisthapteur

Chaque ver possède deux paires d'yeux, situés immédiatement en avant du bord antérieur du pharynx.

L'appareil génital (fig. 7) est du type habituellement observé chez les Caballerocoty!a. Le pore génital commun est situé à gauche, sur la face ventrale, légèrement plus près du pharynx que du bord externe du corps, et à un niveau transversal légèrement antérieur à celui de la constriction pharyngienne. Le pénis est court et ventru (longueur $0,39-0,59 \mathrm{~mm}$, diamètre maximal $0,19-0,25 \mathrm{~mm}$ ) ; le canal déférent est très dilaté sur 


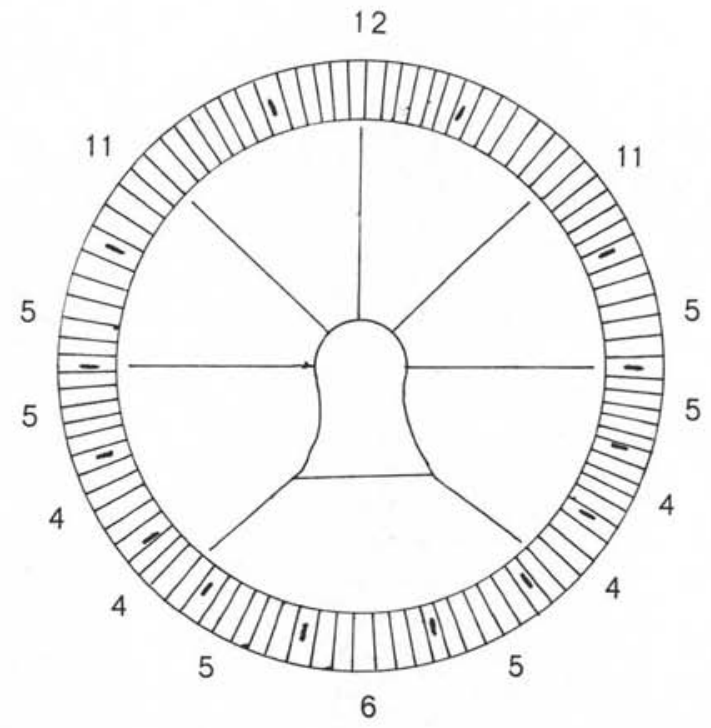

FIG. 4. - Schéma indiquant, sur l'opisthapteur de $C$. verrucosa, la position des petits crochets marginaux par rapport aux festons de la membrane périphérique (avec mention du nombre des festons situés entre deux crochets successifs). Les nombres indiqués peuvent varier de une ou deux unités, et sont souvent légèrement différents d'un côté à l'autre d'un même opisthapteur
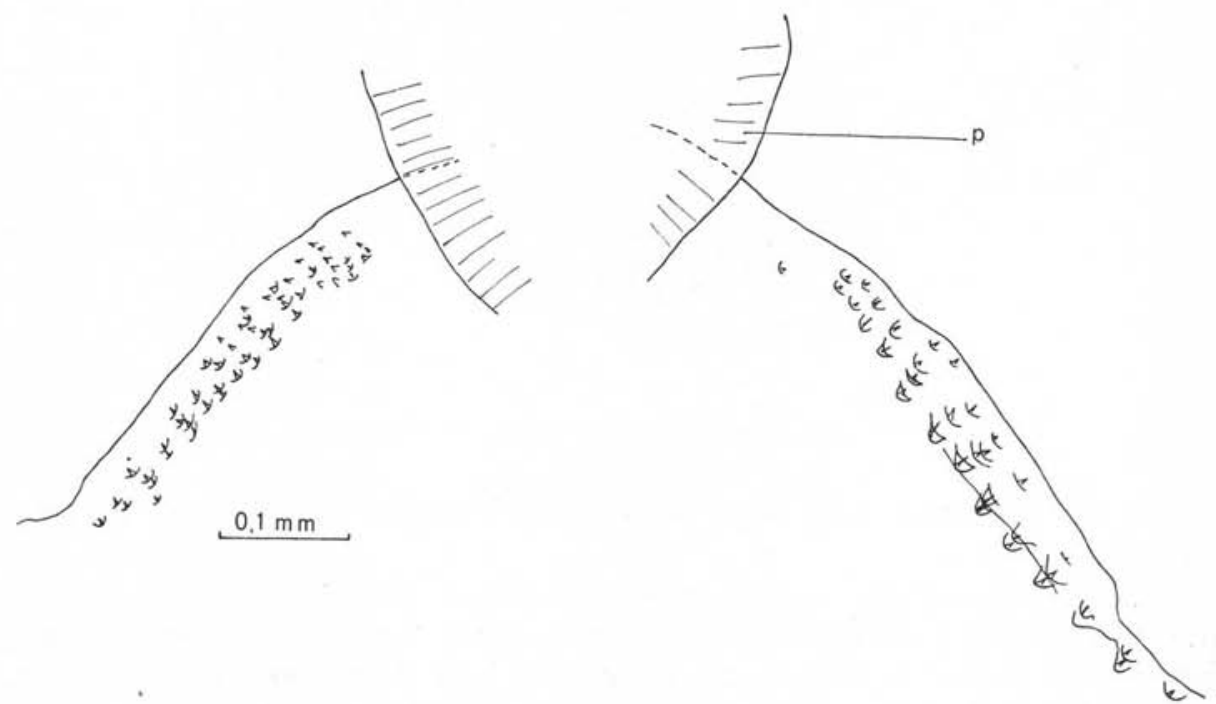

FIG. 5. - Zone des petites épines de la face dorsale de C. verrucosa, vue par transparence à partir de la face ventrale; $p$, prohapteur 


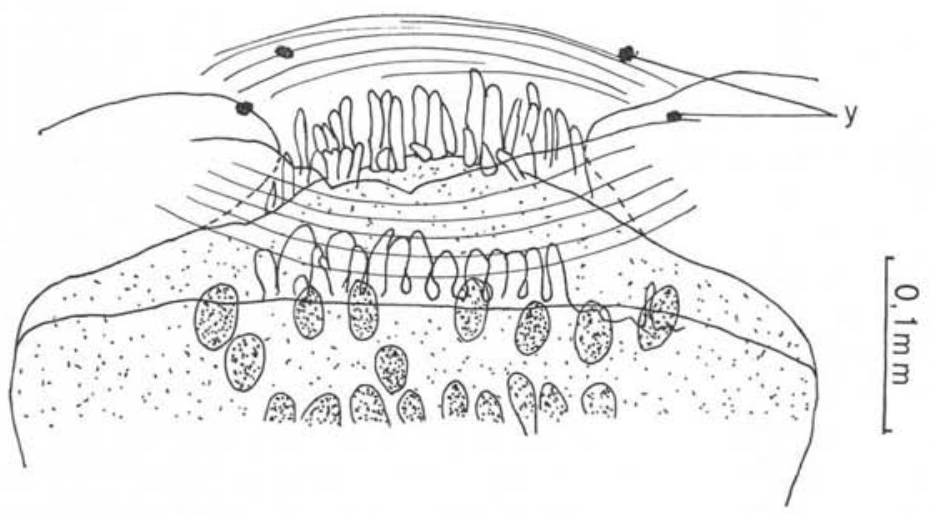

Fig. 6. - Caballerocotyla verrucosa, orifice buccal et partie antérieure du pharynx; $y$, yeux

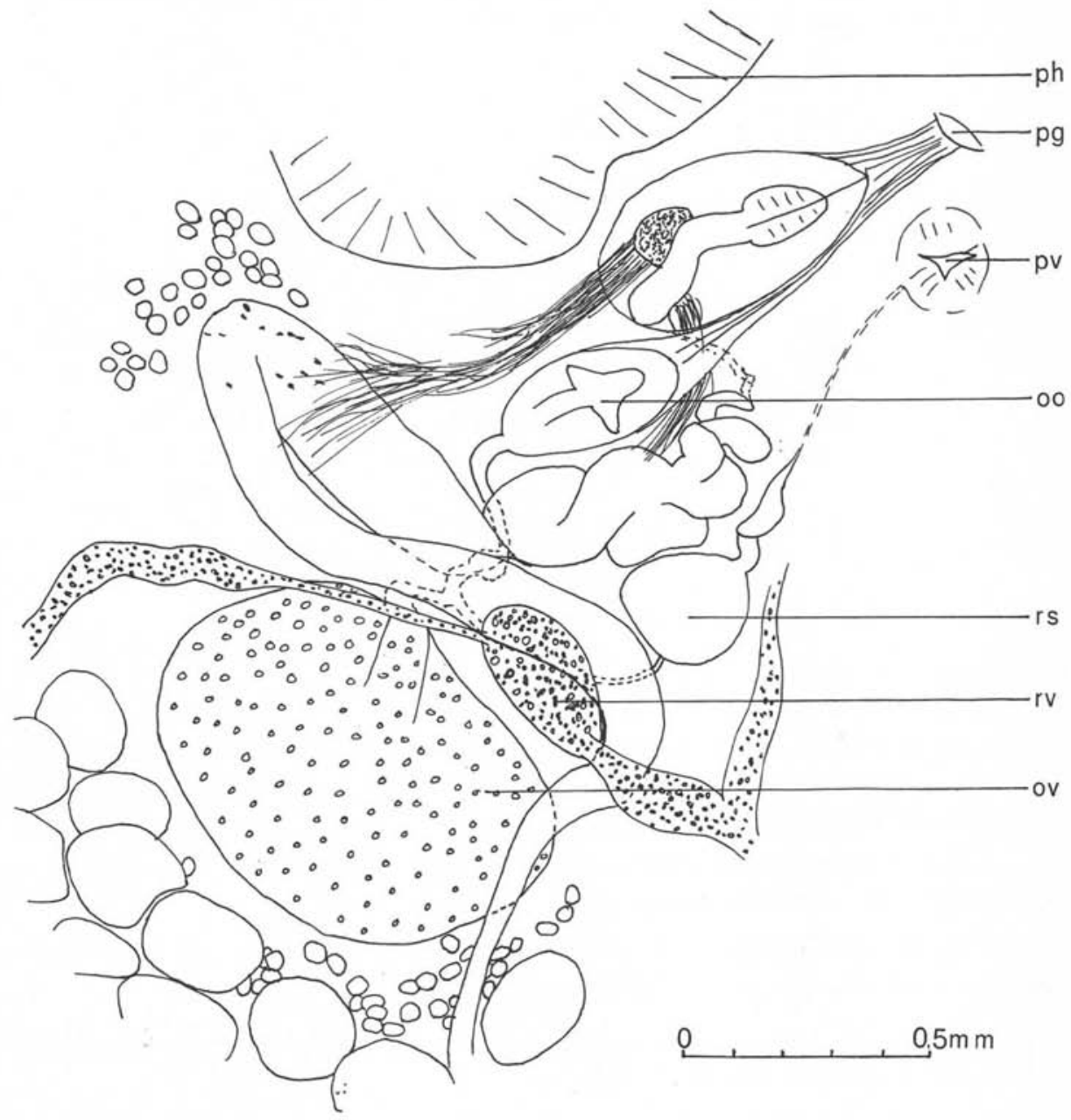

Fig. 7. - Caballerocotyla verrucosa, appareil génital ; oo, ootype; ov, ovaire; pg, pore génital commun ; $p h$, pharynx; $p v$, pore vaginal ; $r s$, réceptacle séminal ; $r v$, réservoir vitellin 
la plus grande partie de son trajet ; il pénètre dans le pénis latéralement, près de la base de celui-ci. Les testicules, très nombreux (plus de 200), ont un diamètre de 0,16 à $0,27 \mathrm{~mm}$; ils sont tous situés dans le champ interintestinal. Utérus, ootype et oviducte ont la disposition habituelle; dans quelques exemplaires seulement l'ootype contient un œuf ; cet œuf (fig. 8) a la forme d'un tétraèdre dont les deux sommets latéraux portent une pointe simple, et le sommet postérieur un prolongement plus complexe; la longueur totale de l'œuf est d'environ $0,12 \mathrm{~mm}$. L'ovaire, ovoïde, mesure de 0,61 à 1,08 sur 0,47 à $0,69 \mathrm{~mm}$; son grand axe est dirigé obliquement en avant vers le côté droit. Les glandes vitellogènes sont réparties dans tout le corps, y compris le champ interintestinal et le lobe céphalique. Le pore vaginal s'ouvre directement en arrière du pore génital commun, à environ $0,20-0,25 \mathrm{~mm}$ de celui-ci ; parfois il paraît entouré d'une formation musculaire à aspect de ventouse; le vagin, plus large et plus sinueux vers l'arrière, s'ouvre dans un réceptacle séminal ovoïde.

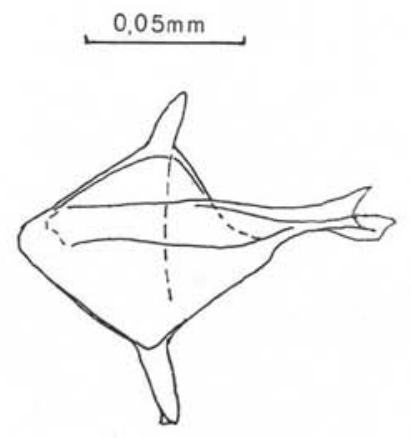

FIG. 8. - Caballerocotyla verrucosa, œuf (observé à l'intérieur de l'ootype)

\section{Discussion :}

Parmi les 13 espèces qui, à notre connaissance, ont été décrites à ce jour dans le genre Caballerocotyla, aucune ne présente ces deux éléments qui nous paraissent tout à fait caractéristiques :

- épines dorsales extrêmement réduites, et limitées à une petite zone faisant immédiatement suite à chaque prohapteur ;

- opisthapteur recouvert sur toute sa face ventrale, y compris sur les côtes, de nombreuses papilles donnant à la ventouse un aspect verruqueux.

C'est pourquoi nous considérons cette espèce comme nouvelle, et nous proposons l'appellation Caballerocotyla verrucosa $\mathrm{n}$. sp. 
CABALLEROCOTYLA ABIDJANI Bussiéras et Baudin-Laurencin, 1970.

Cette espèce est fréquemment observée, fixée à la face interne des opercules de Thunnus albacares; elle n'a jamais été retrouvée chez Thunnus obesus.

A la description précédemment publiée, il convient d'ajouter la présence, tout à fait normale dans le genre Caballerocotyla:

— de deux paires d'yeux, situés immédiatement en avant du bord antérieur du pharynx ;

- de petits crochets marginaux sur l'opisthapteur ; il n'a pas été possible d'observer un opisthapteur portant la totalité des 14 crochets, ceux-ci s'éliminant sans doute plus facilement que chez $C$. verrucosa.

Enfin, l'observation d'un pénis extériorisé a permis de constater :

- que celui-ci est alors couvert de papilles (fig. 9);

- qu'il subit une véritable évagination, puisque sur le pénis rétracté les papilles se situent le long de la lumière médiane.

Ce point est important, car on admet habituellement (cf. notamment Bychowsky 1957) que le pénis des Monogènes présente seulement une translation, sans évagination, ce qui l'opposerait au cirre observé dans divers autres groupes de Plathelminthes.

Par ailleurs, sur un Thunnus albacares ont été isolés quatre spécimens de Caballerocotyla abidjani remarquables par l'extrême réduction de l'opisthapteur (fig. 10).
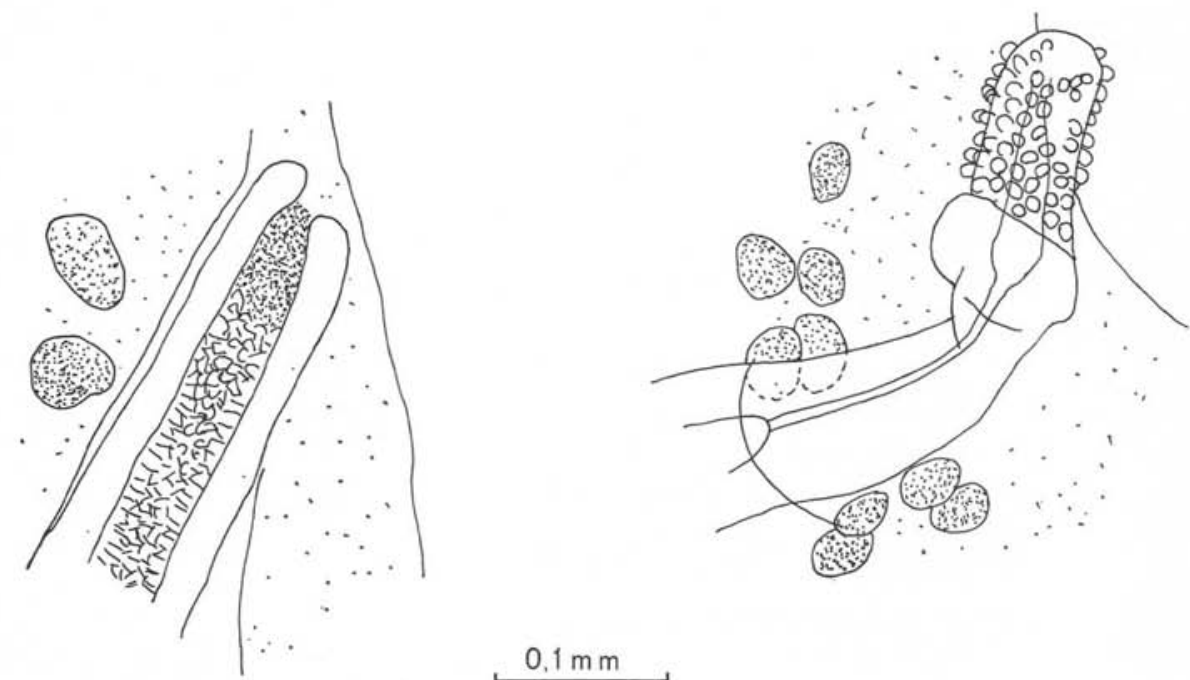

FIG. 9. - Caballerocotyla abidjani, organe copulateur ; à gauche, rétracté ; à droite, protracté; If comparaison des deux dessıns montre nettement que cet organe subit une véritable évagination 


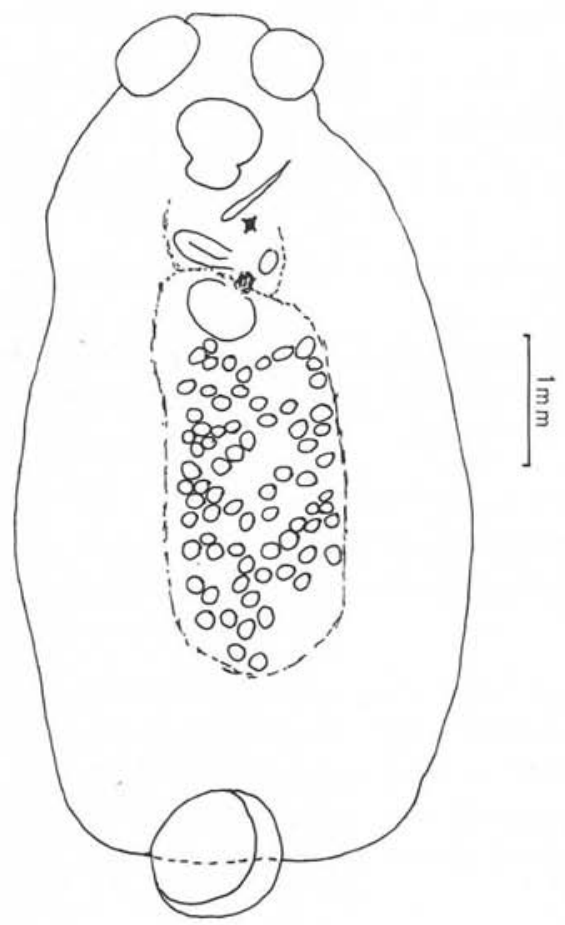

FIG. 10. - Caballerccotyla abidjani microcotyla; remarquer l'extrême atrophie de l'opisthapteur

Mensurations de ces vers :

longueur: $6-8 \mathrm{~mm}$,

largeur: 2,5-3,5 mm.

Prohapteurs :

longueur: $0,63-0,73 \mathrm{~mm}$,

largeur: $0,49-0,53 \mathrm{~mm}$.

Opisthapteur :

longueur : 0,98-1,09 mm,

largeur : $0,88-0,99 \mathrm{~mm}$,

ancres: $68-88 \mu$,

petits crochets marginaux : 11-15 $\mu$,

$\frac{\text { longueur d'un prohapteur }}{\text { longueur de l'opisthapteur }}=0,59-0,66$,

$\frac{\text { largeur de l'opisthapteur }}{\text { largeur du corps }}=0,25-0,39$.

Or ces deux rapports sont, chez les Caballerocotyla abidjani typiaues, respectivement de 0,29-0,37 et de 0,61-0,75. 
Le fait que cette réduction de l'opisthapteur se retrouve sur un lot de quatre spécimens de même origine, et que l'on n'observe pas de formes de transition avec les $C$. abidjani typiques, nous conduit à distinguer dans l'espèce $C$. abidjani deux sous-espèces se définissant ainsi :

Caballerocotyla abidjani abidjani : opisthapteur normalement développé, de diamètre égal à environ les $2 / 3$ de la largeur du corps; le rapport :

$\frac{\text { longueur d'un prohapteur }}{\text { longueur de l'opisthapteur }}$ est toujours très inférieur à 0,5 ; ancres de 93 à $120 \mu$.

Caballerocotyla abidjani microcotyla: opisthapteur très réduit, de diamètre égal à environ $1 / 3$ de la largeur du corps; le rapport: $\frac{\text { longueur d'un prohapteur }}{\text { longueur de l'opisthapteur }}$ est toujours très supérieur à 0,5 ; ancres 68 à $88 \mu$.

\section{CABALLEROCOTYLA PSEUDOMAGRONUM n. sp.}

HôTE :

Thunnus obesus.

LOCALISATION :

Cavité buccale (langue et palais), où persistent des lésions correspondant à l'empreinte de l'opisthapteur, même après l'élimination du ver (fig. 11).

MATÉRIEL ÉTUdiÉ :

13 exemplaires, montés in toto après coloration.

FIG. 11. - Empreinte de l'opisthapteur de Caballerocotyla pseudomagronum sur la langue de Thunnus obesus

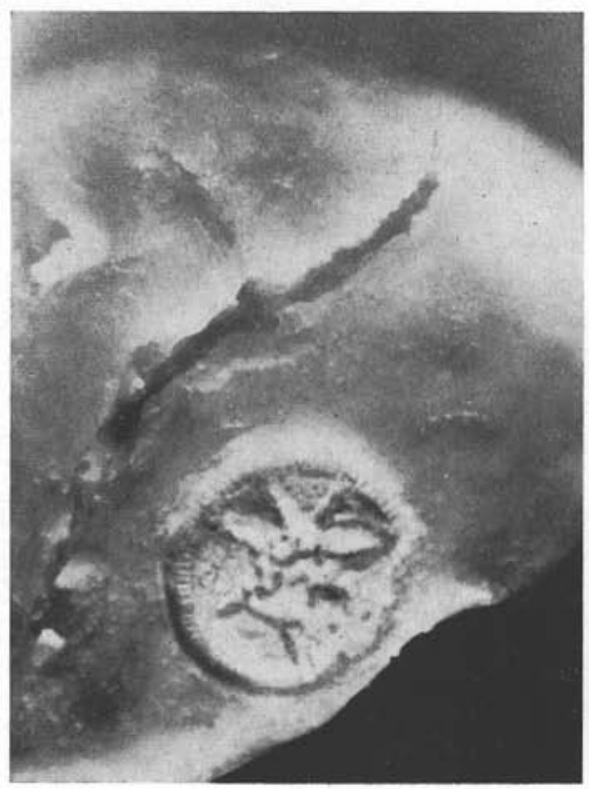




\section{DESCRIPTION :}

Corps ovalaire (fig. 12), 8 - 11 sur $4-7 \mathrm{~mm}$; la plus grande largeur se situe vers la moitié de la longueur; le contour postérieur du corps est arrondi, non échancré.

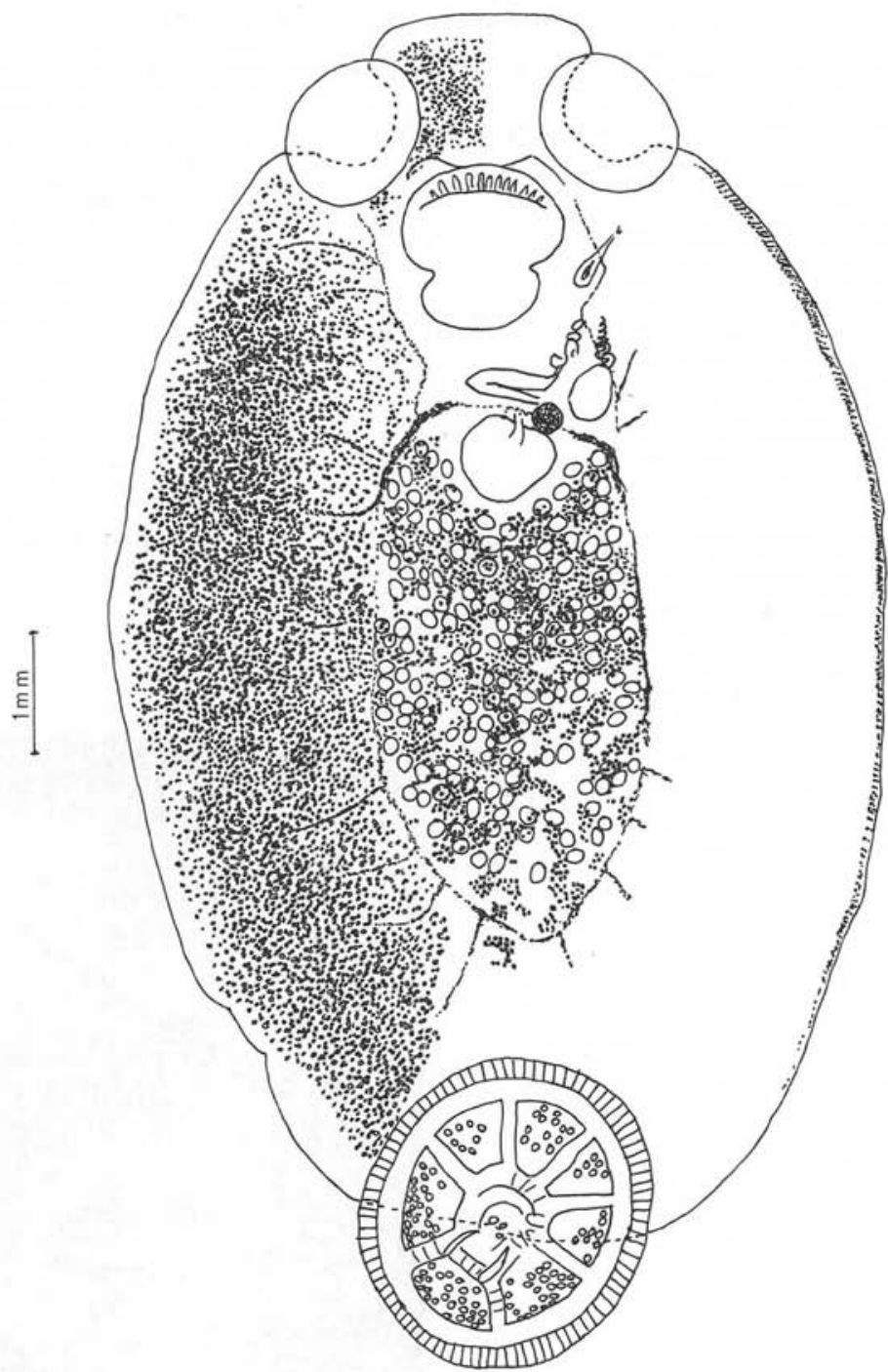

FIG. 12. - Caballerocotyla pseudomagronum, vue ventrale; à gauche de la figure sont représentées les glandes vitellogènes, à droite, les épines marginales dorsales 
En avant, les deux prohapteurs ont une forme ovalaire, grand axe 1,10-1,28 mm, petit axe $0,83-1,04 \mathrm{~mm}$; ils sont distants l'un de l'autre de 0,51 à $0,77 \mathrm{~mm}$.

L'opisthapteur (fig. 13) a un contour légèrement ovalaire; grand axe 1,79-2,25 $\mathrm{mm}$, petit axe 1,66-2,03 $\mathrm{mm}$ (membrane périphérique non comprise). La membrane périphérique mesure de 0,31 à $0,42 \mathrm{~mm}$ de large ; elle est formée d'environ 77 à 80 festons, eux-mêmes portant une étroite bordure frangée (fig. 14). L'heptagone central est de type ouvert, et il en part sept côtes rayonnantes ; on observe quelques papilles circulaires dans les espaces situés entre les côtes, mais non sur les côtes elles-mêmes. Les deux ancres (fig. 15) sont très volumineuses: longueur 0,24 à $0,46 \mathrm{~mm}$, diamètre jusqu'à $70 \mu$; le plus souvent l'ancre gauche est légèrement plus longue que la droite ; chacune paraît généralement formée d'une partie postérieure cylindrique, et d'une partie antérieure conique pointue vers l'avant. L'opisthapteur porte également 14 petits crochets marginaux, disposés régulièrement, et d'une longueur de 18 à $23 \mu$.

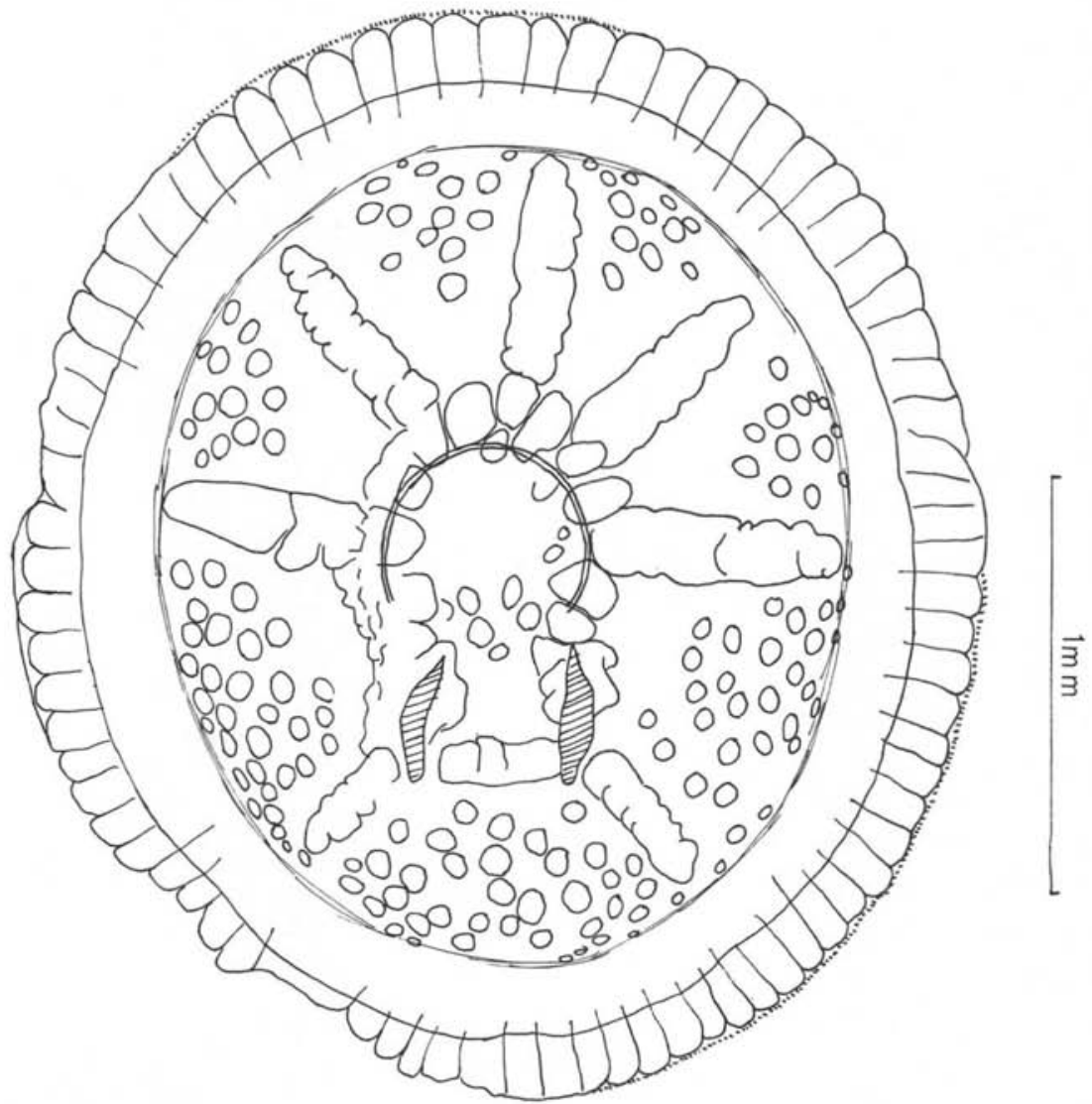

Fic. 13. - Caballerocotyla pseudomagronum, opisthapteur 

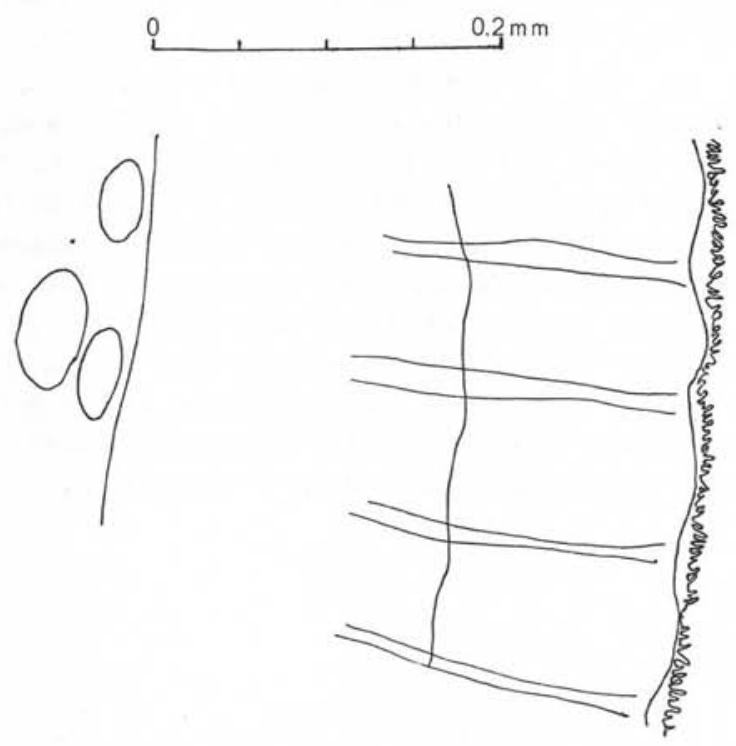

FIG. 14. - C. pseudomagronum, membrane périphérique de l'opisthapteur
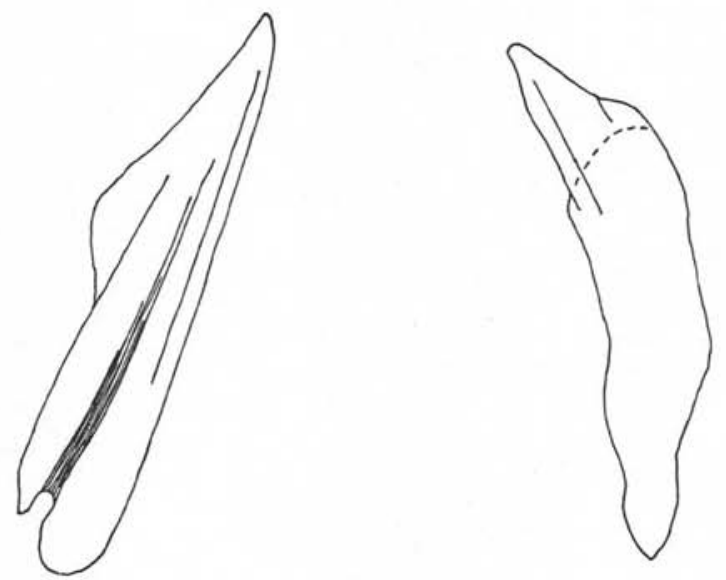

$0,1 \mathrm{~mm}$

Fig. 15. - C. pseudomagronum, ancres de l'opisthapteur, vue dorsale 
Sur la face dorsale du ver se trouvent des épines marginales, à une seule pointe, de 14 à $18 \mu$ de long, disposées en rangées transversales parfois irrégulières (fig. 16) ; ces rangées transversales, très nombreuses, comportent chacune juqu'à sept ou huit épines en région antérieure, mais ce nombre diminue vers l'arrière (trois ou quatre épines par rangée en région médiane, et même disparition totale des épines dans le 1/5 postérieur du corps).

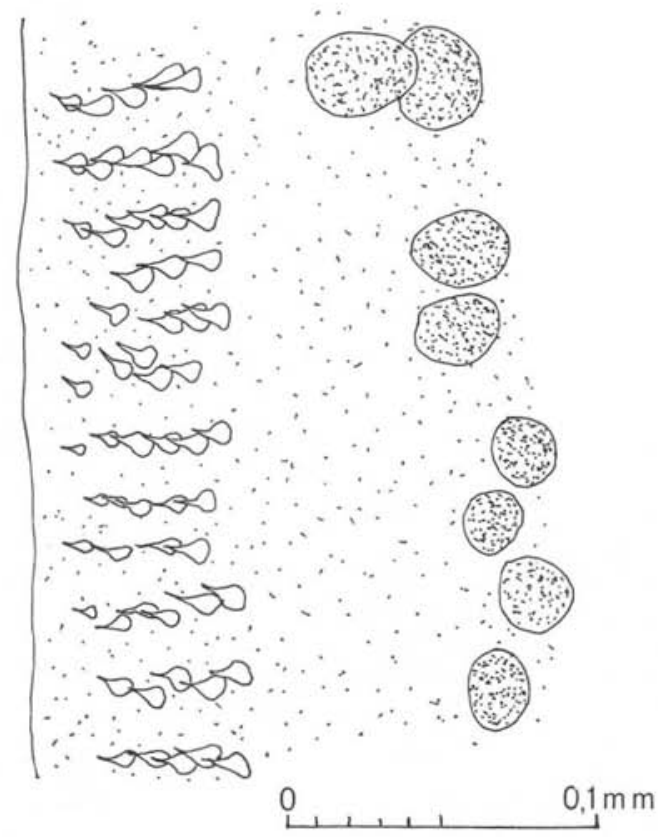

Fig. 16. - C. pseudomagronum, épines marginales dorsales

La bouche se situe à $1,10-1,25 \mathrm{~mm}$ de l'extrémité antérieure du corps ; le pharynx, pourvu d'une constriction, a de 1,10 à $1,58 \mathrm{~mm}$ de long, de 1,16 à 1,42 $\mathrm{mm}$ de large $(0,79$ à $1,05 \mathrm{~mm}$ pour la partie postérieure plus étroite).

Deux paires d'yeux sont observables à l'emplacement habituel, en avant du bord antérieur du pharynx.

L’appareil génital (fig. 17) offre peu de particularités. Le pore génital commun est situé en arrière du prohapteur gauche, au niveau de la plus grande largeur du pharynx, beaucoup plus près de celui-ci que du bord latéral du corps. Le pénis, fusiforme, mesure 0,35 à $0,39 \mathrm{~mm}$ de long, 0,13 à $0,16 \mathrm{~mm}$ de diamètre, son diamètre maximal se trouvant près de sa base ; le pénis est parcouru parallèlement par une vésicule prostatique et par le canal déférent dilaté en une vésicule séminale. Le canal déférent pénètre 


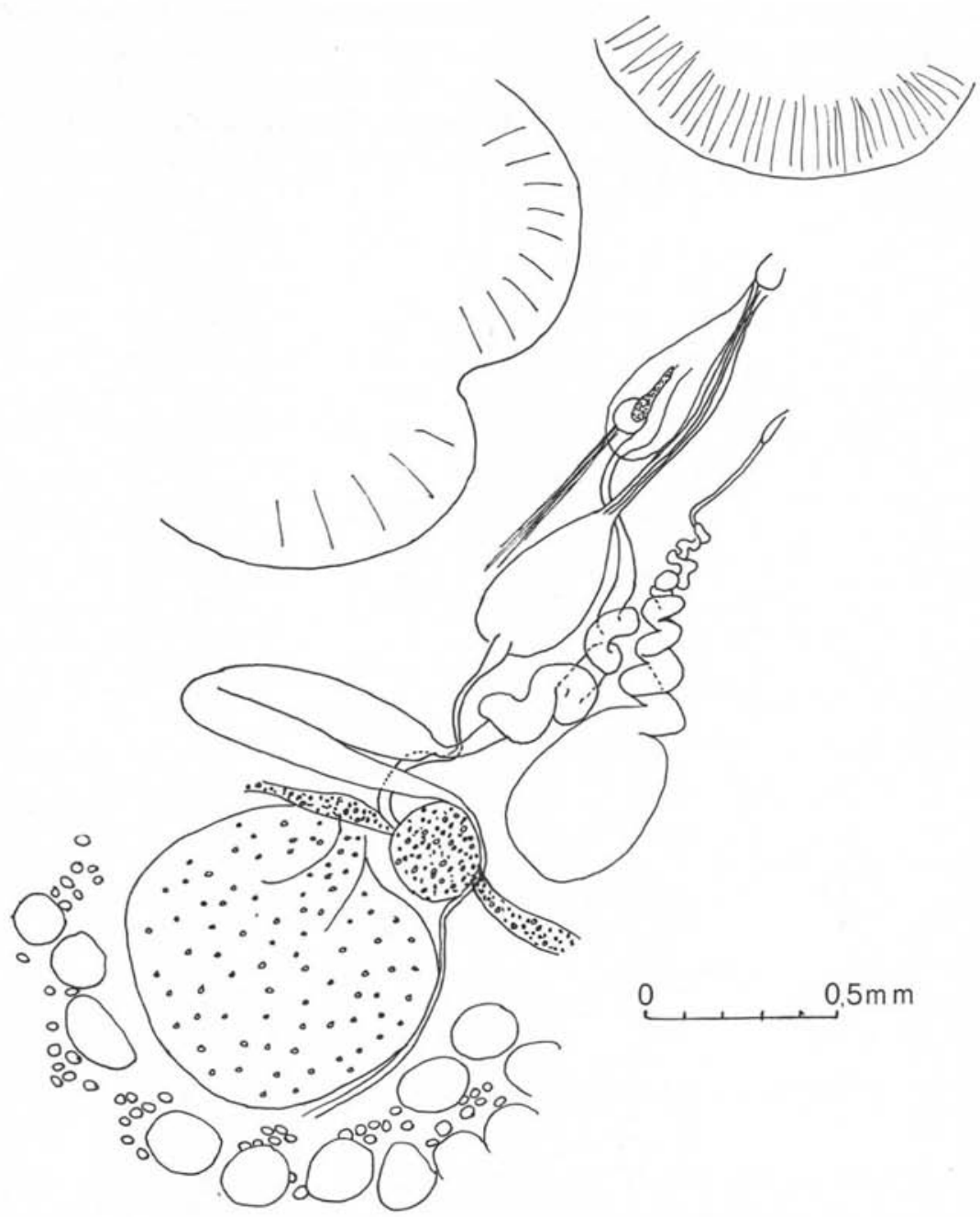

Fig. 17. - C. pseudomagronum, appareil génital

dans le pénis à l'extrémité basale de celui-ci. Les testicules, nombreux (150 à 200), ont un diamètre de 0,12 à $0,18 \mathrm{~mm}$; ils sont tous situés dans le champ interintestinal. Les voies femelles ont la disposition habituelle; l'ovaire est généralement globuleux, presque aussi large que long (grand axe 0,69-0,83 mm, petit axe 0,59-0,79 mm). Les glandes vitellogènes pénètrent dans le lobe céphalique et dans le champ interintestinal. Le pore vaginal s'ouvre en arrière du pore génital commun; le vagin, dans sa moitié postérieure élargie, est disposé en spirale régulière. 


\section{Discussion :}

Ce parasite du patudo se révèle très comparable à Caballerocotyla magronum (Ishii, 1936), parasite des branchies de Thunnus orientalis (Temminck et Schlegel, 1842) au Japon. Ce Capsaliné fut redécrit par Price (1963). Les dimensions de notre espèce sont généralement supérieures, mais de nombreux caractères sont communs en particulier le grand format des ancres et la présence de papilles sur l'opisthapteur. Cependant un élément permet de faire nettement la distinction entre les deux espèces : les épines dorsales de C. magronum, non signalées par Ishii (1936) ni par Ishii et Sawada (1938), sont dites par Price (1963) disposées en trois rangées longitudinales; de plus une illustration de Price montre un système d'épines serrées les unes contre les autres. Au contraire, dans notre espèce, la disposition en rangées transversales est très nette, et il apparaît que, même sur des exemplaires très contractés, aucune confusion ne soit possible.

On peut remarquer en outre que notre espèce parasite la cavité buccale de $T$. obesus, alors que $C$. magronum parasite les branchies de $T$. orientalis, thon qui se rapproche beaucoup plus, parmi les espèces atlantiques, de $T$. thynnus que de $T$. obesus.

Pour ces divers motifs, mais surtout en raison de la différence de disposition des épines dorsales, nous considérons notre espèce comme nouvelle, et nous proposons l'appellation Caballerocotyla pseudomagronum n. sp.

\section{CABALLEROCOTYLA KLAWEI Stunkard, 1962}

Cette espèce, décrite par Stunkard (1962) comme parasite des sacs nasaux de Thunnus macropterus (Temminck et Schlegel, 1842) de l'Océan Pacifique, est régulièrement retrouvée dans les sacs nasaux de Thunnus albacares de l'Atlantique tropical (Bussiéras et Aldrin 1967). On peut d'ailleurs remarquer que ces thons du Pacifique et de l'Atlantique sont souvent considérés comme appartenant à une même espèce, Thunnus albacares (Bonnaterre, 1788).

Aux descriptions déjà publiées de C. klawei, il convient simplement d'ajouter la présence de 14 petits crochets périphériques sur l'opisthapteur. Chaque crochet mesure environ $12 \mu$, et sa pointe passe entre les 2 branches d'une petite pièce accessoire en forme de $\mathrm{V}$, pièce accessoire que l'on retrouve, mais beaucoup moins visible, dans les espèces précédentes de Caballerocotyla.

\section{Répartition par hôte des Capsalinae objets de cette étude.}

\section{1) Thunnus albacares:}

- dans les sacs nasaux : C. klawei,

- sur la face interne des opercules: C. abidjani,

- dans la cavité buccale : $C$. verrucosa, très rarement $C$. abidjani, 
2) Thunnus obesus:

- sur la face interne des opercules: C. verrucosa,

- sur les branchies: très rarement C. verrucosa,

- dans la cavité buccale: C. verrucosa, C. pseudomagronum.

Il est bon de remarquer la différence de localisation de $C$. verrucosa selon qu'il parasite $T$. albacares ou $T$. obesus.

\section{Clé de diagnose des espèces du genre Caballerocotyla}

Une clé de diagnose des espèces du genre Caballerocotyla avait été établie par Price (1960). A la suite de la description de nouvelles espèces, cette clé avait été complétée par Stunkard (1962), Wagner et Carter (1967), Lamothe-Argumedo (1968).

Aussi semble-t-il opportun d'y intégrer les espèces nouvellement décrites, tout en tenant compte de la terminologie actuellement la plus utilisée pour désigner les poissons du groupe des thons et espèces voisines (cf. Collette et Gibbs 1963).

Cela nous conduit à modifier le nom de certains hôtes, en observant les principes suivants :

1) Genre Thunnus South, 1845

A ce genre sont rattachés Neothunnus Kishinouye, 1923, et Parathunnus Kishinouye, 1923.

Thunnus macropterus (Temminck et Schlegel, 1842) du Pacifique, est considéré comme identique à Thunnus albacares (Bonnaterre, 1788) de l'Atlantique, et tombe en synonymie.

Thunnus saliens Jordan et Evermann, 1926, du Pacifique oriental, et Thunnus orientalis (Temminck et Schlegel, 1842) du Pacifique occidental (Japon), sont considérés comme une même sous-espèce du thon rouge Thunnus thynnus (Linné, 1758) : sousespèce Thunnus thynnus orientalis, à répartition indo-pacifique, les populations de thons rouges de l'Atlantique constituant la sous-espèce Thunnus thynnus thynnus.

\section{2) Genre Sarda Cuvier, 1829}

Sarda lineolata (Girard), des côtes de Californie, hôte de Caballerocotyla gregalis, est considéré comme une sous-espèce de Sarda chilensis Cuvier et Valenciennes, 1831.

Sarda orientalis (Temminck et Schlegel, 1842) a été indiqué par Winter (1955) et Price (1963) comme hôte de Caballerocotyla caballerot sur la côte pacifique du Mexique ; cette dénomination sera conservée, bien que Sarda orientalis soit parfois considéré comme limité au Pacifique occidental et à l'Océan Indien, l'espèce des côtes américaines étant alors appelée Sarda velox Meek et Hildebrand, 1923. 
3) Genre Euthynnus Jordan et Gilbert, 1882

L'espèce Thynnus thunnina Cuvier et Valenciennes, 1831, indiquée par Chauhan (1952) comme hôte de Caballerocotyla gouri, doit être dénommée Euthynnus alleteratus (Rafinesque, 1810) ; il convient de ne pas la confondre avec Thynnus thunnina Bleeker, 1850, qui correspond à Euthynnus affinis (Cantor, 1850).

\section{4) Genre Katsuwonus Kishinouye, 1915}

Ce genre, parfois rattaché au précédent, ne comprend qu'une espèce Katsuwonus pelamis (Linné, 1758).

Thynnus vagans Lesson, 1828, devenu Katsuwonus vagans (Lesson, 1828) Jordan, Evermann et Clark, 1930, hôte de Caballerocotyla katsuwoni, tombe en synonymie et doit être dénommé Katsuwonus pelamis.

Sur ces bases, la clé de diagnose du genre Caballerocotyla s'établit ainsi :

1. - Ancres de l'opisthapteur et épines marginales dorsales absentes ; sur les branchies

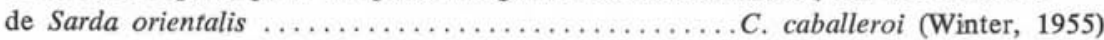
Ancres de l'opisthapteur présentes; épines marginales dorsales présentes ou

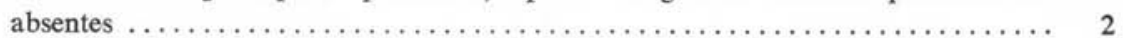

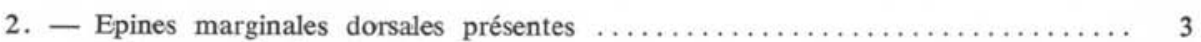

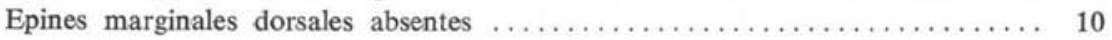

3. - Epines marginales dorsales limitées à une petite zone en arrière des prohapteurs; chez Thunnus albacares et Thunnus obesus (opercules et cavité buccale)

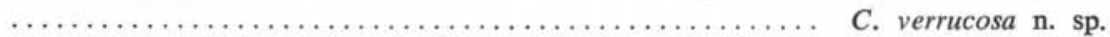
Epines marginales dorsales disposées sur la plus grande partie de la longueur

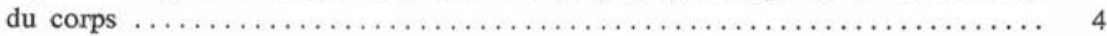

4. - Epines marginales dorsales disposées en nombreuses petites rangées transversales; dans cavité buccale de Thunnus obesus ......... C. pseudomagronum n. sp. Epines marginales dorsales disposées en rangées longitudinales $\ldots \ldots \ldots \ldots \ldots 5$

5. - Epines marginales dorsales en une seule rangée $\ldots \ldots \ldots \ldots \ldots \ldots \ldots \ldots \ldots \ldots$ Epines marginales dorsales en 2 rangées; dans sacs nasaux de Thunnus albacares ............................................. Stunkard, 1962 Epines marginales dorsales en 3 rangées irrégulières, sur les branchies de Thunnus thynnus orientalis ........................ C. magronum (Ishii, 1936)

6. - Epines marginales dorsales avec moins de 12 pointes $\ldots \ldots \ldots \ldots \ldots \ldots \ldots .7$ Epines marginales dorsales avec plus de 12 pointes; sur les opercules de Thunnus albacares ................. C. abidjani Bussiéras et Baudin-Laurencin, 1970

7. - Epines marginales dorsales à 1 pointe; sur les branchies d'Euthynnus allete-

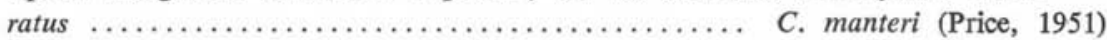
Epines marginales dorsales avec $4-6$ pointes $\ldots \ldots \ldots \ldots \ldots \ldots \ldots \ldots \ldots \ldots .8$ Epines marginales dorsales avec 1-11 pointes; sur les branchies d'Istiophorus greyi ......................... marielenae Lamothe-Argumedo, 1968 
8. - Epines marginales dorsales à 4 pointes; sur des copépodes parasites des branchies de Thunnus albacares ..................... C. biparasitica (Goto, 1894)

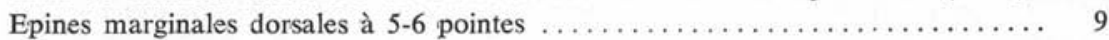

9. - Ancres non recourbées en crochet; sur les opercules d'Euthynnus alleteratus

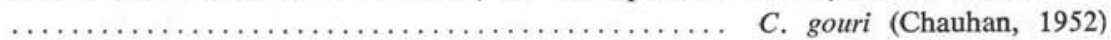
Ancres recourbées en crochet; sur les branchies de Thunnus thynnus orientalis $\ldots \ldots \ldots \ldots \ldots \ldots \ldots \ldots \ldots \ldots \ldots \ldots \ldots \ldots \ldots \ldots$. albsmithi Dollfus, 1962

10. - Papilles marginales présentes; sur les branchies de Sarda sarda ........... $\ldots \ldots \ldots \ldots \ldots \ldots \ldots \ldots \ldots \ldots \ldots \ldots \ldots \ldots \ldots \ldots \ldots \ldots \ldots . \ldots \ldots$. pelamydis (Taschenberg, 1878)

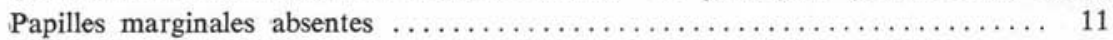

11. - Opisthapteur pédonculé ; sur les branchies de Katsuwonus pelamis .........

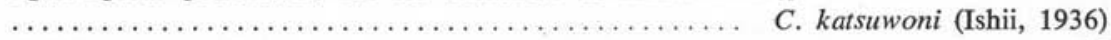

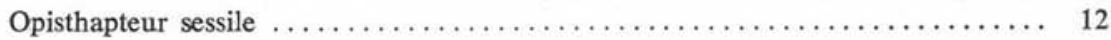

12. - Longueur du corps supérieure à $5 \mathrm{~mm}$; plus de 100 testicules; sur les branchies d'un poisson japonais non identifié (« hazara ») ....... C. foliacea (Goto, 1894) Longueur du corps inférieure à $5 \mathrm{~mm}$; environ 35 testicules; sur les branchies de Sarda chilensis lineolata .............. C. gregalis Wagner et Carter, 1967

\section{Bibliographie}

BussiÉRAS (J.) et ALDRIN (J.-F.), 1967. - Caballerocotyla klawei Stunkard, 1962, Monogène parasite des sacs nasaux du thon albacore de l'Océan Atlantique. Rev. El. Méd. Vét. Pays Trop., 20 (1), 105-108.

- et Baudin-Laurencin (F.), 1970. - Caballerocotyla abidjani n. sp. (Monogenea, Capsalidae), parasite des opercules du thon albacore, Thunnus albacares. Cah. O.R.S.T.O.M., sér. Océanogr., 8 (3), 47-51.

BYCHOWSKY (B. E.), 1957. - Monogenetic Trematodes. Their systematics and phylogeny. Trad. anglaise par Hargis Jr. (W. J.), 1961. Am. Inst. Biol. Sc. Washington.

Chauhan (B. S.), 1952. - Trematodes from Indian marine fishes. Part. VII. On monogenetic parasites of the family Capsalidae, Baird, 1853 (Capsaloidea) from Indian region, with description of a new species of the genus Capsala Bosc, 1811. Rec. Indian Mus., 49 (1), 45-54.

Collette (B. B.) et GibBs Jr. (R. H.), 1963. - Etude préliminaire des poissons de la famille des Scombridae. Actes de la Réunion Scientifique Mondiale sur la biologie du Thon et des espèces voisines, F.A.O., rapports sur les pêches, $\mathrm{n}^{\circ} 6$, vol. 1, 23-32.

IsHII (N.), 1936. - Some new ectoparasitic trematodes of marine fishes (en japonais). Zool. Mag. Tokyo, 48, 783-786.

— et SAwada (T.), 1938. - Studies on the ectoparasitic trematodes. I. Liv. Jub. Travassos, Rio de Janeiro, 231-244. 
Lamothe-Argumedo (R.), 1968. - Monogéneos de peces. VI. Caballerocotyla marielenae sp. nov. (Monogenea: Capsalinae), parasito de las branquias de Istiophorus greyi Jordan and Hill, de Puerto Angel, Oaxaca, México. Riv. di Parassit., 29 (3), 171184.

Price (E. W.), 1960. - The giant marlin, Makiara (sic) marlina Jordan and Evermann, a new host for Capsala pricei Hidalgo, 1959, with a review of the subfamily Capsalinae. Libro Hom. Dr Caballero y Caballero, 237-244.

- 1963. - Redescriptions of some species of Caballerocotyla Price (Monogenea; Capsalidae). Proc. Helm. Soc. Washington, 30 (1), 149-154.

StUNKaRd (H.W.), 1962. - Caballerocotyla klawei sp.n., a Monogenetic Trematode from the nasal capsule of Neothunnus macropterus. J. of Parasit., 48 (6), 883-890.

WAGNER (E. D.) et CARTER (C. E.), 1967. - Caballerocotyla gregalis sp.n. (Trematoda: Monogenea) from the gills of Sarda lineolata (Girard). J. of Parasit., 53 (2), 277279.

Winter (H. A.), 1955. - Capsala caballeroi sp. n., parasito de Sarda orientalis, con un catálogo de los trematodos monogeneos de los peces del Océano Pacifico de las Américas. Rev. Brazil. Biol., 15 (1), 9,32. 